\title{
Conductance oscillations in strongly correlated fractional quantum Hall line junctions
}

\author{
U. Zülicke \\ Institut für Theoretische Festkörperphysik, Universität Karlsruhe, D-76128 Karlsruhe, Germany and \\ Institute of Fundamental Sciences, Massey University, Private Bag 11 222, Palmerston North, New Zealand* \\ E. Shimshoni \\ Department of Mathematics-Physics, University of Haifa at Oranim, Tivon 36006, Israel
}

(Dated: February 2, 2008)

\begin{abstract}
We present a detailed theory of transport through line junctions formed by counterpropagating single-branch fractional-quantum-Hall edge channels having different filling factors. Intriguing transport properties are exhibited when strong Coulomb interactions between electrons from the two edges are present. Such strongly correlated line junctions can be classified according to the value of an effective line-junction filling factor $\tilde{\nu}$ that is the inverse of an even integer. Interactions turn out to affect transport most importantly for $\tilde{\nu}=1 / 2$ and $\tilde{\nu}=1 / 4$. A particularly interesting case is $\tilde{\nu}=1 / 4$ corresponding to, e.g., a junction of edge channels having filling factor 1 and $1 / 5$, respectively. We predict its differential tunneling conductance to oscillate as a function of voltage. This behavior directly reflects the existence of novel Majorana-fermion quasiparticle excitations in this type of line junction. Experimental accessibility of such systems in current cleaved-edge overgrown samples enables direct testing of our theoretical predictions.
\end{abstract}

\section{INTRODUCTION}

One-dimensional (1D) electron systems ${ }^{1}$ have long been the focus of theoretical and experimental research. Initially, theorists studied them as rare examples of exactly soluble interacting many-body systems ${ }^{2.3 .4}$ They served as a basis for the development of powerful new theoretical tools such as bosonization ${ }^{5,6,7}$ and refermionization ${ }^{8}$ techniques. Due to their intriguing non-Fermi-liquid properties, interacting 1D electron systems are classified within the distinct phenomenology of Luttinger-liquid behavior ${ }^{9}$ Eventually, realizations of quasi-1D electron systems were found in metallic materials with strongly anisotropic resistivity ${ }^{10}$ Recent fabrication of clean long semiconductor quantum wires 11 as well as carbon nanotubes ${ }^{12}$ created new possibilities to observe Luttinger-liquid behavior in experiment.

An especially versatile type of 1D electron system is realized at the boundary of two-dimensional (2D) electron systems in a strong perpendicular magnetic field. At particular values of the filling factor $\nu=2 \pi \ell^{2} n_{2 \mathrm{D}}$, where $\ell=$ $\sqrt{\hbar c /|e B|}$ is the magnetic length and $n_{2 \mathrm{D}}$ the electron sheet density, the $2 \mathrm{D}$ system becomes incompressible in the bulk, ${ }^{13}$ giving rise to quantized values of the Hall resistance. In this regime where the quantum Hall $(\mathrm{QH})$ effect $^{14.15}$ is observed, low-lying excitations exist at the sample boundary ${ }^{16}$ whose electronic properties are analogous to chiral versions of Luttinger liquids when the filling factor at the incompressibility is fractional. ${ }^{17}$ Unlike the more conventional types of quasi-1D metals, the properties of edge excitations in a $\mathrm{QH}$ sample can be easily tailored. Simple adjustment of the magnetic field can create different QH states in the bulk of the 2D system with concomitant change in the edge's chiral-Luttinger-liquid properties. Advanced nanostructuring techniques enable the creation of novel tunneling geometries ${ }^{18,19,20,21}$ involving $\mathrm{QH}$ edges as well as line junctions between them. 22,23,24 While line junctions between counterpropagating chiral edge channels having the same integer filling factor closely mirror properties of conventional quasi-1D systems, ${ }^{25,26,27}$ an entirely new arena for novel correlation effects is opened up when the edge channels forming the junction belong to fractional-QH samples. For the case of a disordered junction, these have been investigated in Refs. [28 29]. A related study for a clean system of tunnel-coupled copropagating fractional-QH edge channels in bilayers was performed recently. . $^{30.31}$

Here we consider the situation where the single-branch edge channels forming the line junction have opposite chirality and belong to $\mathrm{QH}$ systems with fractional filling factors $1 /\left(m_{1}+1\right)$ and $1 /\left(m_{2}+1\right)$ with even integers $m_{1} \neq m_{2}$. We assume the junction region to be clean and of finite length $L$, having edge-channel leads attached that contact to four reservoirs where transport measurements can be performed. This sample geometry is experimentally realizable in recently grown corner junctions between mutually orthogonal 2D electron systems ${ }^{21.24}$ To enable tunneling transport between them, the two edge channels have to be close enough in space, which typically facilitates strong interchannel Coulomb interactions within the junction region. This turns out to significantly affect the junction conductance when the effective filling factor $\tilde{\nu}=1 /\left|m_{1}-m_{2}\right|$ is equal to $1 / 2$ or $1 / 4$. Using bosonization and refermionization techniques, we succeed for both cases in mapping the originally strongly interacting linejunction system onto a system of noninteracting fermions. For $\tilde{\nu}=1 / 2$, these new quasiparticles are fictitious chiral spin$1 / 2$ fermions which have no direct physical meaning. Only observables related to their pseudo-spin degree of freedom correspond to measurable quantities. In the other case of $\tilde{\nu}=1 / 4$, two Majorana fermions having different velocities $v_{+}$and $v_{-}$turn out to be the fundamental particle excitations in the line junction. The existence of a velocity splitting directly results in oscillations of the differential junction conductance as a function of the transport voltage, which can be detected experimentally. Its observation would confirm the existence of yet another type of exotic quasiparticle in lowdimensional systems.

This article is organized as follows. We start, in Sec. II by specifying the model for an isolated $\mathrm{QH}$ line junction and 
apply the techniques of bosonization and refermionization to obtain its solution. The emergence of new quasiparticles will be elucidated. In the following Sec. III the coupling of such a line junction to external edge-channel leads is considered. We derive relations between the chemical potentials in the leads to quantities describing the line junction that take full account of charging effects. These results are then applied in Sec. IV to calculate transport properties of line junctions, with particular focus on the cases $\tilde{\nu}=1 / 2$ and $\tilde{\nu}=1 / 4$. A summary and conclusions are presented in Sec. $\mathrm{V}$ This article provides full details of and extends results reported in a previous short publication 32

\section{EFFECTIVE LOW-ENERGY MODEL FOR A QUANTUM-HALL LINE JUNCTION}

A single branch of low-lying edge excitations exists in QH samples at the Laughlin series of filling factors $\nu_{m}=$ $1 /(m+1)$. These form chiral 1D electron systems that can be described, ${ }^{17}$ using the bosonization ${ }^{33}$ approach, by a single chiral boson field $\phi_{m}(x)$. The (suitably normal-ordered) electronic charge density at a location $x$ along the edge is given by $\varrho_{m}(x)=: \psi_{m}^{\dagger}(x) \psi_{m}(x):=\sqrt{\nu_{m}} \partial_{x} \phi_{m}(x) /(2 \pi)$. Its dynamics is determined by the Hamiltonian

$$
H_{m}=\frac{\hbar v_{m}}{4 \pi} \int d x\left(\partial_{x} \phi_{m}\right)^{2} .
$$

The edge velocity $v_{m}$ is the sum of a one-electron contribution $v_{\mathrm{F}}$, which is proportional to the slope of the external potential confining electrons in the $\mathrm{QH}$ sample, and an interaction contribution $\nu_{m} U /(2 \pi \hbar)$ that typically dominates in the long-wave-length limit ${ }^{34}$ The chirality of a $\mathrm{QH}$ edge is manifested by the fact that disturbances in the electronic charge density propagate only in one particular direction along the sample perimeter. Mathematically, this is expressed by the canonical commutation relations $\left[\phi_{m}(x), \phi_{m}\left(x^{\prime}\right)\right]=$ $i \chi \pi \operatorname{sgn}\left(x-x^{\prime}\right)$ for the boson field $\phi_{m}$. The chirality parameter $\chi$ assumes the value $+1(-1)$ for right(left)-movers. An especially useful property of chiral $1 \mathrm{D}$ systems is the complete equivalence of their descriptions in terms of bosonic and fermionic degrees of freedom ${ }^{8.35}$ For single-branch $\mathrm{QH}$ edges, this is expressed by the bosonization identity ${ }^{17}$ of the electron annihilation operator,

$$
\psi_{m}(x)=\sqrt{z_{m}} \mathcal{F}_{m} \exp \left\{i x \frac{Y}{\ell^{2}}+i \chi \frac{\phi_{m}(x)}{\sqrt{\nu_{m}}}\right\} .
$$

Here $z_{m}$ is a normalization constant, and $Y$ denotes the guiding-center location of electrons at the Fermi energy. The Klein factor $\mathcal{F}_{m}$ acts as a ladder operator for the electron number and ensures fermionic statistics of bosonized electron operators from different $\mathrm{QH}$ edges or edge branches. ${ }^{8}$

We are interested in studying a $\mathrm{QH}$ line junction that is formed when two parallel fractional-QH edge channels with opposite chirality are coupled by uniform tunneling along a finite length $L$. Such a junction can be realized, e.g., by fabricating two 2D electron systems that are laterally separated $^{22}$ or form a corner junction. ${ }^{21,24}$ In the junction region, the charge densities and, hence, respective bosonic fields $\phi_{m_{1}}$ and $\phi_{m_{2}}$ describing the two edges are coupled via interactions and tunneling. The total Hamiltonian of the line junction is thus given by $H_{\mathrm{J}}=H_{\mathrm{LL}}+H_{\text {tun }}$, where

$$
\begin{aligned}
& H_{\mathrm{LL}}=H_{m_{1}}+H_{m_{2}}+H_{\mathrm{int}}, \\
& H_{\mathrm{int}}=\frac{\sqrt{\nu_{m_{1}} \nu_{m_{2}}}}{4 \pi^{2}} U \int_{-\frac{L}{2}}^{\frac{L}{2}} d x \partial_{x} \phi_{m_{1}} \partial_{x} \phi_{m_{2}}, \\
& H_{\text {tun }}=\int_{-\frac{L}{2}}^{\frac{L}{2}} d x\left\{t \psi_{m_{1}}^{\dagger} \psi_{m_{2}}+\text { H.c. }\right\} .
\end{aligned}
$$

Here we have assumed equal strengths for intra- and interchannel interactions which is the case for typical line junctions.

The part $H_{\mathrm{LL}}$ of the line-junction Hamiltonian can be diagonalized in a straightforward manner. For the case $m_{1}=m_{2}$, the familiar phase-field description ${ }^{9}$ of a nonchiral Luttinger liquid is recovered in the typical situation where the Coulomb matrix element dominates the bare velocities $v_{\mathrm{F} j}(j=1,2)$. Addition of the tunneling term $H_{\text {tun }}$ in its bosonized form yields an orthodox sine-Gordon model whose properties have been studied extensively ${ }^{36}$ We do not discuss this nonchiral case here any further. Instead, we focus on situations where $m_{1} \neq m_{2}$. Then the line junction is intrinsically chiral, also in the limit of strong Coulomb interactions ${ }^{51}$ Instead, we can write $H_{\mathrm{LL}}$ as a sum of independent contributions from two chiral normal modes,

$$
H_{\mathrm{LL}}=\frac{\hbar}{4 \pi} \int_{-\frac{L}{2}}^{\frac{L}{2}} d x\left\{v_{\mathrm{a}}\left(\partial_{x} \phi_{\mathrm{a}}\right)^{2}+v_{\mathrm{b}}\left(\partial_{x} \phi_{\mathrm{b}}\right)^{2}\right\} .
$$

The normal-mode boson fields $\phi_{\mathrm{a}}$ and $\phi_{\mathrm{b}}$ obey the commutation relations $\left[\phi_{\mathrm{a}, \mathrm{b}}(x), \phi_{\mathrm{a}, \mathrm{b}}\left(x^{\prime}\right)\right]=i \chi_{\mathrm{a}, \mathrm{b}} \pi \operatorname{sgn}\left(x-x^{\prime}\right)$ with $\chi_{\mathrm{a}}=-\chi_{\mathrm{b}}$. Assuming the realistic limit where the bare edge velocities are much smaller than the Coulomb matrix element, we find the expressions

$$
\begin{aligned}
v_{\mathrm{a}} & =\frac{\left|\nu_{m 1}-\nu_{m 2}\right| U}{2 \pi \hbar}+\frac{\nu_{m_{1}} v_{\mathrm{F} 1}+\nu_{m_{2}} v_{\mathrm{F} 2}}{\left|\nu_{m 1}-\nu_{m 2}\right|} \\
v_{\mathrm{b}} & =\frac{\nu_{m_{2}} v_{\mathrm{F} 1}+\nu_{m_{1}} v_{\mathrm{F} 2}}{\left|\nu_{m 1}-\nu_{m 2}\right|} \\
\chi_{\mathrm{b}} & =-\chi_{1} \operatorname{sgn}\left(\nu_{m_{1}}-\nu_{m_{2}}\right) \\
\phi_{\mathrm{a}} & =\frac{\left(\sqrt{\nu_{m_{1}}}-\varepsilon \sqrt{\nu_{m_{2}}}\right) \phi_{m_{1}}+\left(\sqrt{\nu_{m_{2}}}-\varepsilon \sqrt{\nu_{m_{1}}}\right) \phi_{m_{2}}}{\sqrt{\left|\nu_{m_{1}}-\nu_{m_{2}}\right|}}(5, \mathrm{~d}) \\
\phi_{\mathrm{b}} & =\frac{\left(\sqrt{\nu_{m_{2}}}+\varepsilon \sqrt{\nu_{m_{1}}}\right) \phi_{m_{1}}+\left(\sqrt{\nu_{m_{1}}}+\varepsilon \sqrt{\nu_{m_{2}}}\right) \phi_{m_{2}}(5 . \mathrm{e})}{\sqrt{\left|\nu_{m_{1}}-\nu_{m_{2}}\right|}}
\end{aligned}
$$

Here we have defined a small parameter (of order $\hbar v_{\mathrm{F} j} / U$ )

$$
\varepsilon=\frac{\sqrt{\nu_{m_{1}} \nu_{m_{2}}}}{\left|\nu_{m_{1}}-\nu_{m_{2}}\right|} \frac{v_{\mathrm{F} 1}+v_{\mathrm{F} 2}}{v_{\mathrm{a}}}
$$

and neglected terms of quadratic order in $\varepsilon^{52}$

After bosonization, the tunneling Hamiltonian reads

$$
H_{\text {tun }}=2|t| \sqrt{z_{m_{1}} z_{m_{2}}} \int_{-\frac{L}{2}}^{\frac{L}{2}} d x \cos \left(\frac{\phi_{\mathrm{n}}}{\sqrt{\tilde{\nu}}}+x \frac{\Delta}{\ell^{2}}\right),
$$


where we have absorbed the phase of the tunneling matrix element into the neutral-mode bosonic field

$$
\phi_{\mathrm{n}}=\frac{\sqrt{\nu_{m_{2}}} \phi_{m_{1}}+\sqrt{\nu_{m_{1}}} \phi_{m_{2}}}{\sqrt{\left|\nu_{m_{1}}-\nu_{m_{2}}\right|}}-\chi_{1} \sqrt{\tilde{\nu}} \arg (t)
$$

The abbreviation $\tilde{\nu}=\nu_{m_{1}} \nu_{m_{2}} /\left|\nu_{m_{1}}-\nu_{m_{2}}\right| \equiv 1 /\left|m_{1}-m_{2}\right|$ has the meaning of an effective junction filling factor, and $\Delta=Y_{1}-Y_{2}$ is a measure for the width of the line junction. To first order in small quantities defined above, the neutral mode is given in terms of the normal modes of $H_{\mathrm{LL}}$ as $\phi_{\mathrm{n}}=\phi_{\mathrm{b}}-\varepsilon \phi_{\mathrm{a}}$. (Here we have used the freedom to redefine the normal-mode fields by a constant shift to absorb the phase of the tunneling matrix element in $\phi_{\mathrm{b}}$.) If we furthermore assume weak enough tunneling such that $t \ell \ll U$, then terms in $H_{\text {tun }}$ that couple the normal modes of $H_{\mathrm{LL}}$ are of second

order in small quantities. As we will see below, this is generally the relevant physical situation and even required in the case $\tilde{\nu}=1 / 4$. In what follows we restrict ourselves to the approximation $\varepsilon \rightarrow 0$ (corresponding to $U \rightarrow \infty$ ). We note, however, that corrections to leading order in $\varepsilon$ can be included perturbatively, yielding small corrections to our final results which do not affect them significantly. The Hamiltonian of the line junction is then diagonalized, to a good approximation, by the normal modes $\phi_{\mathrm{a}}$ and $\phi_{\mathrm{b}}$ of $H_{\mathrm{LL}}$, and we find $H_{\mathrm{J}}=H_{\mathrm{a}}+H_{\mathrm{b}}^{\left(\frac{1}{\bar{\nu}}\right)}$, where

$$
H_{\mathrm{a}}=\frac{\hbar v_{\mathrm{a}}}{4 \pi} \int_{-\frac{L}{2}}^{\frac{L}{2}} d x\left(\partial_{x} \phi_{\mathrm{a}}\right)^{2}
$$

$$
H_{\mathrm{b}}^{\left(\frac{1}{\tilde{\nu}}\right)}=\int_{-\frac{L}{2}}^{\frac{L}{2}} d x\left\{\frac{\hbar v_{\mathrm{b}}}{4 \pi}\left(\partial_{x} \phi_{\mathrm{b}}\right)^{2}+2|t| \sqrt{z_{m_{1}} z_{m_{2}}} \cos \left(\frac{\phi_{\mathrm{b}}}{\sqrt{\tilde{\nu}}}+x \frac{\Delta}{\ell^{2}}\right)\right\} .
$$

The fast normal mode, labeled a, turns out to be free and unaffected by tunneling. This is quite clear physically, as this mode is closely related to the total charge density in the line junction $\left(\rho_{\mathrm{a}} \equiv \frac{\partial_{x} \phi_{\mathrm{a}}}{2 \pi}\right)$, which is left invariant in any tunneling process. As we are only interested in studying tunneling transport, we do not consider the fast mode any further. The slow mode, being approximately equal to the neutral mode which measures the difference in electron densities for the two edge branches forming the junction, has a dynamics that is strongly influenced by the tunneling term. Its Hamiltonian $H_{\mathrm{b}}^{\left(\frac{1}{\nu}\right)}$ is that of a chiral sine-Gordon model which has been studied in different contexts before ${ }^{30.37}$ Such theories can be classified according to the different values of the effective junction filling factor $\tilde{\nu}$ that is given here by the inverse of even integers. For $1 / \tilde{\nu}>4$, the cosine term has been shown ${ }^{30}$ to be irrelevant in a renormalization-group sense. This means that it does not alter the excitation spectrum of the free chiral boson theory in any important way and can therefore be treated as a perturbation. The situations when $1 / \tilde{\nu}=4$ and 2 , however, turn out to be different. While no perturbative approach is permissible for these cases, the effect of the cosine term can nevertheless be calculated, even exactly, using bosonization identities of the kind expressed in Eq. (2). Namely, it is possible ${ }^{30.37}$ to map the rather complicated chiral bosonic field theory for the slow mode onto that of noninteracting fictitious fermions. We proceed to show this in the following two subsections, as the refermionized description of the slow-mode dynamics forms the basis for our subsequent transport calculations.

\section{A. Case $\tilde{\nu}=1 / 2$ : Fictitious chiral fermion tunneling}

To solve the chiral sine-Gordon Hamiltonian with $\tilde{\nu}=1 / 2$, we introduce - for purely mathematical reasons - a ghost field $\eta(x)$ that has the same chirality and dynamics as the slow mode $\phi_{\mathrm{b}}$. It is then possible to define a pair of fictitious chiral fermions, distinguished by a pseudospin degree of freedom $\sigma=\uparrow, \downarrow$ using the bosonization identity

$$
\Psi_{\sigma}(x)=\sqrt{z_{\mathrm{b}}} \mathcal{F}_{\sigma} \exp \left\{i \chi_{\mathrm{b}} \frac{\eta(x)+\sigma \phi_{\mathrm{b}}(x)}{\sqrt{2}}\right\} .
$$

In this new notation, the Hamiltonian $H_{\mathrm{b}}^{(2)}$ of the slow mode represents tunneling between the two flavors of fictitious fermions:

$$
H_{\mathrm{b}}^{(2)}=\int_{-\frac{L}{2}}^{\frac{L}{2}} d x\left\{\hbar v_{\mathrm{b}} \sum_{\sigma} \Psi_{\sigma}^{\dagger}\left(-i \chi_{\mathrm{b}} \partial_{x}\right) \Psi_{\sigma}+\tilde{t}_{m_{1} m_{2}}\left[\Psi_{\uparrow}^{\dagger} \Psi_{\downarrow} e^{-i \chi_{\mathrm{b}} x \Delta / \ell^{2}}+\text { H.c. }\right]\right\}
$$

The tunneling strength $\tilde{t}_{m_{1} m_{2}}=|t| \sqrt{z_{m_{1}} z_{m_{2}}} / z_{\mathrm{b}}$ is generally different, because of chiral-Luttinger-liquid properties, from the matrix element for tunneling between the original linejunction edge channels ${ }^{53}$ Physical observables are expressed 
in terms of pseudospin-related quantities, while the charge degree of freedom for fictitious fermions remains hidden from measurement. For example, the spin density and the density associated with the slow mode are equivalent:

$$
\Psi_{\uparrow}^{\dagger} \Psi_{\uparrow}-\Psi_{\downarrow}^{\dagger} \Psi_{\downarrow}=\sqrt{2} \varrho_{\mathrm{b}} \equiv \frac{\partial_{x} \phi_{\mathrm{b}}}{\sqrt{2} \pi}
$$

The representation of $H_{\mathrm{b}}^{(2)}$ in terms of the fictitious chiral pseudo-spin-1/2 fermion, being quadratic in this field, makes it possible to treat transport straightforwardly. This will be discussed below in SectionIV A

\section{B. Case $\tilde{\nu}=1 / 4$ : Velocity-split Majorana fermions}

A bosonization identity of the type given in Eq. (2) can be used to define a Dirac fermion in terms of the chiral boson field $\phi_{\mathrm{b}}$ :

$$
\psi_{\mathrm{b}}(x)=\sqrt{z_{\mathrm{b}}} \mathcal{F}_{\mathrm{b}} \exp \left\{i \chi_{\mathrm{b}}\left[x \frac{\Delta}{2 \ell^{2}}+\phi_{\mathrm{b}}(x)\right]\right\} .
$$

The density $\varrho_{\mathrm{b}}$ of the slow mode is related to the normalordered density : $\psi_{\mathrm{b}}^{\dagger} \psi_{\mathrm{b}}$ : of the new fictitious fermion via

$$
\varrho_{\mathrm{b}} \equiv \frac{\partial_{x} \phi_{\mathrm{b}}}{2 \pi}=: \psi_{\mathrm{b}}^{\dagger} \psi_{\mathrm{b}}:-\frac{\Delta}{2 \ell^{2}}
$$

With the help of the relation ${ }^{30}$

$$
\psi_{\mathrm{b}} i \partial_{x} \psi_{\mathrm{b}}=-2 \pi \chi_{\mathrm{b}} z_{\mathrm{b}}^{2} \mathcal{F}_{\mathrm{b}}^{2} \exp \left\{i \chi_{\mathrm{b}}\left[2 \phi_{\mathrm{b}}+x \frac{\Delta}{\ell^{2}}\right]\right\}
$$

the Hamiltonian of the slow mode can be rewritten as

$$
H_{\mathrm{b}}^{(4)}=\int_{-\frac{L}{2}}^{\frac{L}{2}} d x\left\{\hbar v_{\mathrm{b}} \psi_{\mathrm{b}}^{\dagger}\left(-i \chi_{b} \partial_{x}\right) \psi_{\mathrm{b}}-\hbar v_{\mathrm{b}} \frac{\Delta}{2 \ell^{2}} \psi_{\mathrm{b}}^{\dagger} \psi_{\mathrm{b}}+\frac{\hbar v_{\mathrm{t}}}{2}\left[\psi_{\mathrm{b}}\left(-i \chi_{\mathrm{b}} \partial_{x}\right) \psi_{\mathrm{b}}+\psi_{\mathrm{b}}^{\dagger}\left(-i \chi_{\mathrm{b}} \partial_{x}\right) \psi_{\mathrm{b}}^{\dagger}\right]\right\}
$$

where the tunneling matrix element has been absorbed into the velocity parameter $v_{\mathrm{t}}=|t| \sqrt{z_{m_{1}} z_{m_{2}}} / \pi \hbar z_{\mathrm{b}}^{2}$. In the representation of the fictitious fermion $\psi_{\mathrm{b}}, H_{\mathrm{b}}^{(4)}$ looks like the Bogoliubov-de Gennes Hamiltonian ${ }^{38}$ of a spinless $\mathrm{p}-$ wave superconductor. It differs from similar systems considered previously 39,40 by its chiral 1D nature. We do not explicitly pursue the superconducting analogy here any further (although the formalism employed below could be phrased within such a framework). Instead, we use the fact that the real and imaginary parts of a Dirac fermion are Majorana fermions and define fields $\xi_{ \pm} \equiv \xi_{ \pm}^{\dagger}$ via

$$
\begin{aligned}
& \xi_{+}=\frac{\psi_{\mathrm{b}}+\psi_{\mathrm{b}}^{\dagger}}{\sqrt{2}}, \\
& \xi_{-}=\frac{\psi_{\mathrm{b}}-\psi_{\mathrm{b}}^{\dagger}}{\sqrt{2} i} .
\end{aligned}
$$

Note that $: \psi_{\mathrm{b}}^{\dagger} \psi_{\mathrm{b}}:=i \xi_{+} \xi_{-}$. The expression for the Hamiltonian of the slow mode reads

$H_{\mathrm{b}}^{(4)}=\int_{-\frac{L}{2}}^{\frac{L}{2}} d x\left\{\frac{\hbar}{2} \sum_{r= \pm} v_{r} \xi_{r}\left(-i \chi_{\mathrm{b}} \partial_{x}\right) \xi_{r}-i \frac{\hbar v_{\mathrm{b}} \Delta}{\ell^{2}} \xi_{+} \xi_{-}\right\}$

with different velocities $v_{r}=v_{\mathrm{b}}+r v_{\mathrm{t}}$ for the two Majorana fields. Hence, while the slow mode is characterized by a single velocity $v_{\mathrm{b}}$, tunneling leads to the generation of two different velocities for the resulting quasiparticles that turn out to be Majorana fermions. A dynamically generated velocity splitting of this type has been found before in tunnelcoupled interacting quantum wires ${ }^{41,42,43}$ and quantum-Hall bilayers. ${ }^{30}$ It is reminiscent of the spin-charge separation expected to occur in interacting $1 \mathrm{D}$ electron systems. 1 The representation of $H_{\mathrm{b}}^{(4)}$ in terms of the fictitious noninteracting Majorana fermions enables treatment of transport through the line junction, which will be shown below in Sec.IVB Diagonalization of $H_{\mathrm{b}}^{(4)}$ is straightforward. Its spectrum has two branches,

$$
E_{k, \pm}=\hbar \chi_{\mathrm{b}} k\left(v_{\mathrm{b}} \pm v_{\mathrm{t}} \sqrt{1+r_{k}^{2}}\right)
$$

with $r_{k}=v_{\mathrm{b}} \Delta /\left(v_{\mathrm{t}} k \ell^{2}\right)$. The corresponding eigenstates are given, using a spinor notation in the basis of Majorana fermions $\xi_{ \pm}$, as

$$
\begin{aligned}
& \left(\begin{array}{l}
\xi_{+} \\
\xi_{-}
\end{array}\right)_{+}=\left(\begin{array}{c}
c_{k} \\
i \chi_{\mathrm{b}} s_{k}
\end{array}\right) e^{i k x} \\
& \left(\begin{array}{c}
\xi_{+} \\
\xi_{-}
\end{array}\right)_{-}=\left(\begin{array}{c}
i \chi_{\mathrm{b}} s_{k} \\
c_{k}
\end{array}\right) e^{i k x}
\end{aligned}
$$

Here we used the abbreviations

$$
\left.\begin{array}{l}
c_{k} \\
s_{k}
\end{array}\right\}=\frac{\sqrt{\sqrt{1+r_{k}^{2}} \pm 1}}{\sqrt{2 \sqrt{1+r_{k}^{2}}}} .
$$

\section{TUNNELING CURRENT, CHEMICAL POTENTIAL, AND COUPLING TO VOLTAGES}

To be able to calculate conductances, it is necessary to treat the nonequilibrium situation where a finite voltage is applied 
to the line junction. This requires proper definition of operators for currents and chemical potentials within the junction region, which we set out to do in the first part of this section. Our results are then applied to relate these quantities to externally adjustable lead voltages.

A standard calculation yields the expression for the tunneling current flowing from edge channel 1 to edge channel 2 within the line junction as

$$
I_{\mathrm{J}}=\frac{i}{\hbar} \int_{-\frac{L}{2}}^{\frac{L}{2}} d x\left\{t \psi_{m_{1}}^{\dagger} \psi_{m_{2}}-\text { H.c. }\right\}
$$

After bosonization, and within the same approximations used above, i.e., for small $\varepsilon$ [see Eq. [6], we find the following expression for the transport current through the line junction:

$$
I_{\mathrm{J}}^{\left(\frac{1}{\tilde{\nu}}\right)}=\chi_{1} \frac{2|t|}{\hbar} \sqrt{z_{m_{1}} z_{m_{2}}} \int_{-\frac{L}{2}}^{\frac{L}{2}} d x \sin \left(\frac{\phi_{\mathrm{b}}}{\sqrt{\tilde{\nu}}}+x \frac{\Delta}{\ell^{2}}\right)
$$

It comes as no surprise that the current, as the tunneling Hamiltonian, depends only on the slow normal mode. Hence, only the latter features in our transport calculation. Defining the spatially varying partial current

$$
I_{\mathrm{b}}^{\left(\frac{1}{\tilde{\nu}}\right)}(x)=\chi_{1} \frac{|t|}{\hbar \sqrt{\tilde{\nu}}} \sqrt{z_{m_{1}} z_{m_{2}}} \int_{-\frac{L}{2}}^{\frac{L}{2}} d x^{\prime} \sin \left(\frac{\phi_{\mathrm{b}}\left(x^{\prime}\right)}{\sqrt{\tilde{\nu}}}+x^{\prime} \frac{\Delta}{\ell^{2}}\right) \operatorname{sgn}\left(x-x^{\prime}\right)
$$

turns out to be useful for later. Obviously, $I_{\mathrm{b}}^{\left(\frac{1}{\bar{v}}\right)}(L / 2)=$ $-I_{\mathrm{b}}^{\left(\frac{1}{\tilde{\nu}}\right)}(-L / 2) \equiv I_{\mathrm{J}}^{\left(\frac{1}{\tilde{\nu}}\right)} /(2 \sqrt{\tilde{\nu}})$.

The local chemical potential of the slow normal mode can be defined in the usual way ${ }^{44}$ as the functional derivative of the Hamiltonian with respect to density: $\mu_{\mathrm{b}}^{(\mathrm{J})}(x)=\delta H_{\mathrm{J}} / \delta \varrho_{\mathrm{b}}(x)$. Using the commutation relations for chiral boson fields, it is straightforward to prove that $\chi_{\mathrm{b}} \phi_{\mathrm{b}}$ is canonically conjugate to $\varrho_{\mathrm{b}}$, and therefore

$$
\frac{\delta \mathcal{O}}{\delta \rho_{\mathrm{b}}(x)}=i \chi_{\mathrm{b}}\left[\phi_{\mathrm{b}}(x), \mathcal{O}\right]
$$

for an arbitrary functional $\mathcal{O}$. Application of this identity yields an expression for the local chemical potential,

$$
\mu_{\mathrm{b}}^{(\mathrm{J})}(x)=2 \pi \hbar\left\{v_{\mathrm{b}} \varrho_{\mathrm{b}}(x)-\chi_{1} I_{\mathrm{b}}^{\left(\frac{1}{\nu}\right)}(x)\right\} .
$$

As a special case of this equation, we find the chemical potentials at the endpoints of the junction to be

$$
\mu_{\mathrm{b}}^{(\mathrm{J})}\left( \pm \frac{L}{2}\right)=2 \pi \hbar\left\{v_{\mathrm{b}} \varrho_{\mathrm{b}}\left( \pm \frac{L}{2}\right) \mp \frac{\chi_{1} I_{\mathrm{J}}^{\left(\frac{1}{\tilde{\nu}}\right)}}{2 \sqrt{\tilde{\nu}}}\right\} .
$$

The continuity equation for the slow-mode density can be derived in a similar way,

$$
\begin{aligned}
\frac{d}{d \tau} \varrho_{\mathrm{b}} & =\partial_{\tau} \varrho_{\mathrm{b}}+\frac{i}{\hbar}\left[H_{\mathrm{J}}, \varrho_{\mathrm{b}}\right] \\
& =\partial_{\tau} \varrho_{\mathrm{b}}+\chi_{b} \partial_{x}\left\{v_{\mathrm{b}} \varrho_{\mathrm{b}}-\chi_{1} I_{\mathrm{b}}^{\left(\frac{1}{\bar{\nu}}\right)}\right\} .
\end{aligned}
$$

In the stationary limit where $\partial_{\tau} \varrho_{\mathrm{b}}=0$, Eqs. 26) and 28b imply the exact relation

$$
\partial_{x} \mu_{\mathrm{b}}^{(\mathrm{J})}=2 \pi \hbar \chi_{\mathrm{b}} \frac{d}{d \tau} \varrho_{\mathrm{b}}
$$

Integrating it, and observing the relation $\sqrt{\tilde{\nu}} \frac{d}{d \tau} \int_{x} \varrho_{\mathrm{b}}=$ $I_{\mathrm{J}}^{\left(\frac{1}{\tilde{\nu}}\right)} \operatorname{sgn}\left(\nu_{m_{1}}-\nu_{m_{2}}\right)$, we find

$$
\mu_{\mathrm{b}}^{(\mathrm{J})}\left(\frac{L}{2}\right)-\mu_{\mathrm{b}}^{(\mathrm{J})}\left(-\frac{L}{2}\right)=-2 \pi \hbar \chi_{1} \frac{I_{\mathrm{J}}^{\left(\frac{1}{\tilde{\nu}}\right)}}{\sqrt{\tilde{\nu}}}
$$

and comparison with Eqs. 27] yields

$$
\varrho_{\mathrm{b}}\left(\frac{L}{2}\right)=\varrho_{\mathrm{b}}\left(-\frac{L}{2}\right) \equiv \bar{\varrho}_{\mathrm{b}}
$$

The periodic boundary condition expressed in Eq. 31 is a nontrivial property of the stationary state that enables us to treat the line junction as separate from any edge-channel leads that couple it to external reservoirs. The effect of external voltages will be to set the appropriate value of $\bar{\varrho}_{\mathrm{b}}$ consistent with the value $I_{\mathrm{J}}$ for the line-junction current. A derivation of these relations will be presented in the following paragraphs.

Edge channels forming the line junction typically exist beyond the junction region but are not coupled anymore via tunneling or interactions. These incoming and outgoing edge branches serve as noninteracting leads that couple the junction to external reservoirs with experimentally controllable chemical potentials. (See the figure in Ref. [32] and Fig. 11 below for illustration.) The Hamiltonian for the lead regions $|x|>L / 2$ is therefore given by that of two uncoupled edges; $H_{\mathrm{E}}=H_{m_{1}}+H_{m_{2}}$. As the separate chiral edge densities for each edge branch are just linear combinations of the densities $\varrho_{\mathrm{a}}$ and $\varrho_{\mathrm{b}}$ that are the normal modes in the junction, we can write down an expression for $H_{\mathrm{E}}$ in terms of the latter. In the limit of strong intra-edge Coulomb interactions, which is the physically relevant situation that we have been considering all along, it reads 


$$
H_{\mathrm{E}}=\frac{U}{2\left|\nu_{m_{1}}-\nu_{m_{2}}\right|} \int_{|x|>\frac{L}{2}} d x\left\{\left(\nu_{m_{1}}^{2}+\nu_{m_{2}}^{2}\right) \varrho_{\mathrm{a}}^{2}+2 \nu_{m_{1}} \nu_{m_{2}} \varrho_{\mathrm{b}}^{2}-2 \sqrt{\nu_{m_{1}} \nu_{m_{2}}}\left(\nu_{m_{1}}+\nu_{m_{2}}\right) \varrho_{\mathrm{a}} \varrho_{\mathrm{b}}\right\} \quad .
$$

While the a and $\mathrm{b}$ modes are certainly not normal modes in the lead region, it is nevertheless possible to define their chemical potentials via $\mu_{\mathrm{a}, \mathrm{b}}^{(\mathrm{E})}(x)=\delta H_{\mathrm{E}} / \delta \varrho_{\mathrm{a}, \mathrm{b}}(x)$. As $H_{\mathrm{E}}$ is a nondiagonal quadratic form when expressed in terms of $\varrho_{\mathrm{a}}$ and $\varrho_{\mathrm{b}}$, each of the chemical potentials $\mu_{\mathrm{a}}^{(\mathrm{E})}$ and $\mu_{\mathrm{b}}^{(\mathrm{E})}$ depends on both densities. In other words, there exists a finite cross-capacitance between the $\mathrm{a}$ and $\mathrm{b}$ modes in the lead region. Re-expressing $\mu_{\mathrm{a}, \mathrm{b}}$ in terms of the original chiral edge densities, we find

$$
\begin{aligned}
\frac{\mu_{\mathrm{a}}^{(\mathrm{E})}}{U} & =\frac{\nu_{m_{1}} \varrho_{m_{1}}-\nu_{m_{2}} \varrho_{m_{2}}}{\sqrt{\left|\nu_{m_{1}}-\nu_{m_{2}}\right|}} \operatorname{sgn}\left(\nu_{m_{1}}-\nu_{m_{2}}\right), \\
\frac{\mu_{\mathrm{b}}^{(\mathrm{E})}}{U} & =-\sqrt{\tilde{\nu}}\left(\varrho_{m_{1}}-\varrho_{m_{2}}\right) \operatorname{sgn}\left(\nu_{m_{1}}-\nu_{m_{2}}\right),
\end{aligned}
$$

where again terms of order $\varepsilon$ have been neglected. The expressions given in Eq. (33) are useful because the local chemical potential in each of the chiral edge branches within the lead region is directly related to the local chiral edge density in that branch via $\mu_{j}=U \varrho_{j}$, within the limit of strong intraedge Coulomb interactions considered here. Experimentally, two of the four lead branches' chemical potentials are con-
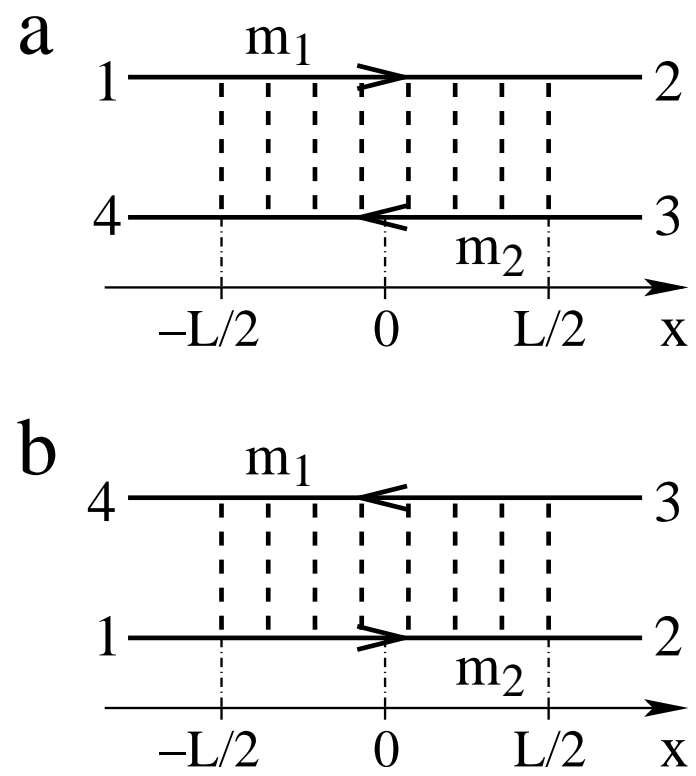

FIG. 1: Line junction attached to chiral edge-channel leads. The chemical potentials of incoming lead branches (1 and 3) are fixed experimentally by external reservoirs. These, together with the tunneling current in the junction region (defined to flow from the $m_{1}$ branch to the $m_{2}$ branch and indicated by vertical dashed lines), determine the boundary condition for the slow normal-mode density at the junction boundaries $x= \pm L / 2$. Panels a and $\mathrm{b}$ show the two possible chiralities of the junction that can be distinguished, e.g., by the chirality of the $m_{1}$ branch: $\chi_{1}=+1(-1)$ for a (b). trolled by attachment to external reservoirs, namely those having chiral edge-density waves propagating away from a reservoir. We adopt the convention that the incoming (outgoing) edge lead for $x<-L / 2$ is labeled 1 (4), whereas the incoming (outgoing) edge lead for $x>L / 2$ is labeled 3 (2). (See Fig.11) We find then from Eqs. (33)

$$
\left.\mu_{\mathrm{b}}^{(\mathrm{E})}\right|_{x>\mp \frac{L}{2}}=\chi_{\mathrm{b}} \sqrt{\tilde{\nu}}\left(\mu_{1 / 2}-\mu_{4 / 3}\right) .
$$

Requiring continuity of the chemical potentials $\mu_{\mathrm{a}, \mathrm{b}}$ at the lead-junction interfaces, and using Eqs. (27), 31) and (5c) as well as the constancy of $\mu_{\mathrm{a}}$ throughout the line junction, we find the relations

$$
\begin{aligned}
4 \pi \hbar \chi_{\mathrm{b}} v_{\mathrm{b}} \bar{\varrho}_{\mathrm{b}} & =\sqrt{\tilde{\nu}}\left(\mu_{1}-\mu_{3}+\mu_{2}-\mu_{4}\right), \\
\frac{2 \pi \hbar I_{\mathrm{J}}^{\left(\frac{1}{\nu}\right)}}{\operatorname{sgn}\left(\nu_{m_{1}}-\nu_{m_{2}}\right)} & =-\tilde{\nu}\left(\mu_{1}+\mu_{3}-\mu_{2}-\mu_{4}\right), \\
\nu_{m_{1}}\left(\mu_{1(4)}-\mu_{2(3)}\right) & =\nu_{m_{2}}\left(\mu_{4(1)}-\mu_{3(2)}\right) .
\end{aligned}
$$

Here we used a compact notation in Eq. (35c) to show the cases corresponding to both line-junction chiralities $\chi_{1}=$ $+(-) 1$ in one line. Straightforward elimination yields an equation for the voltage drop across the junction,

$$
\frac{\mu_{1}-\mu_{3}}{2 \pi \hbar}=\frac{\chi_{\mathrm{b}}}{\sqrt{\tilde{\nu}}} v_{\mathrm{b}} \bar{\varrho}_{\mathrm{b}}+\chi_{1} \frac{\nu_{m_{1}}+\nu_{m_{2}}}{2 \nu_{m_{1}} \nu_{m_{2}}} I_{\mathrm{J}}^{\left(\frac{1}{\tilde{\nu}}\right)}
$$

providing a link between the externally applied voltage $\mathrm{eV}=$ $\mu_{1}-\mu_{3}$ and intrinsic line-junction quantities, such as the tunneling current and the boundary value $\bar{\varrho}_{\mathrm{b}}$ of the slow-mode density. This is one of the central results of our work presented here. In deriving Eq. [36), we have fully taken into account charging of the line junction arising from its coupling to leads. Solving it together with the intrinsic dynamics of the slow mode (induced by its Hamiltonian $H_{\mathrm{b}}^{\left(\frac{1}{\nu}\right)}$ ) completely determines the current.

\section{RESULTS FOR TRANSPORT THROUGH LINE JUNCTIONS}

We have seen, in Sec. III that the tunneling current through the line junction is determined by the dynamics of the slow mode only. Relating its Hamiltonian to that of free quasiparticles, which are Dirac fermions for $\tilde{\nu}=1 / 2$ and Majorana fermions for $\tilde{\nu}=1 / 4$, we are able to calculate its dynamics exactly. With the help of Eq. [36, quantities determining this dynamics are related to the externally applied voltage. Pulling both understandings together, we find the line-junction conductance. As it is quite different for the two cases mentioned above, we discuss them in separate subsections. 


\section{A. Case $\tilde{\nu}=1 / 2$ : Conductance oscillations as a function of junction length}

Within the refermionization procedure applied in Sec. IIA we find the current through the junction as the tunneling current between the two flavors of a fictitious chiral Dirac pseudo-spin 1/2 fermion:

$I_{\mathrm{J}}^{(2)}=-i \chi_{\mathrm{b}} \chi_{1} \frac{\tilde{t}_{m_{1} m_{2}}}{\hbar} \int_{-\frac{L}{2}}^{\frac{L}{2}} d x\left\{\Psi_{\uparrow}^{\dagger} \Psi_{\downarrow} e^{-i \chi_{\mathrm{b}} x \Delta / \ell^{2}}-\right.$ H.c. $\}$

The chemical-potential difference between the $\uparrow$ and $\downarrow$ branches is related to that of the slow mode via

$$
\mu_{\uparrow}-\mu_{\downarrow}=\sqrt{2} \mu_{\mathrm{b}}^{(\mathrm{J})} .
$$

Equation (31), together with Eq. (12), implies a constant density difference between the two fermion flavors along the junction. This provides the driving force for the current.

The chiral-tunneling problem can be solved exactly using standard methods ${ }^{45,46.47}$ Here we employ the scattering approach to transport. ${ }^{48.49}$ Using an obvious spinor notation for the pseudospin-1/2 fermion wave functions, the following Ansätze for eigenstates of the Hamiltonian $H_{\mathrm{b}}^{(2)}$ [displayed in Eq. [11] in the three regions $\chi_{\mathrm{b}} x<-L / 2, \chi_{\mathrm{b}} x>L / 2$, and $|x|<L / 2$ can be written down:

$$
\begin{aligned}
& \left.\left(\begin{array}{c}
\Psi_{\uparrow} \\
\Psi_{\downarrow}
\end{array}\right)\right|_{\chi_{\mathrm{b}} x<-\frac{L}{2}}=\left(\begin{array}{c}
1 \\
0
\end{array}\right) e^{i x \frac{\chi_{\mathrm{b}} E}{\hbar v_{\mathrm{b}}}} \\
& \left.\left(\begin{array}{c}
\Psi_{\uparrow} \\
\Psi_{\downarrow}
\end{array}\right)\right|_{\chi_{\mathrm{b}} x>\frac{L}{2}}=\left(\begin{array}{c}
t_{\uparrow} \\
0
\end{array}\right) e^{i x \frac{\chi_{\mathrm{b}} E}{\hbar v_{\mathrm{b}}}}+\left(\begin{array}{c}
0 \\
t_{\downarrow}
\end{array}\right) e^{i x \frac{\chi_{\mathrm{b}} E}{\hbar v_{\mathrm{b}}}} \\
& \left.\left(\begin{array}{c}
\Psi_{\uparrow} \\
\Psi_{\downarrow}
\end{array}\right)\right|_{|x|<\frac{L}{2}}=a\left(\begin{array}{c}
d_{+} \\
d_{-}
\end{array}\right) e^{i x \kappa_{+}}+b\left(\begin{array}{c}
-d_{-}^{*} \\
d_{+}^{*}
\end{array}\right) e^{i x}
\end{aligned}
$$

with the abbreviations

$$
\begin{aligned}
& d_{ \pm}= \pm \sqrt{\sqrt{1+\zeta^{2}} \pm \zeta} e^{\mp i \chi_{\mathrm{b}} x \frac{\Delta}{2 \ell^{2}}}, \\
& \kappa_{ \pm}=\chi_{\mathrm{b}} \frac{E \pm \tilde{t}_{m_{1} m_{2}} \sqrt{1+\zeta^{2}}}{\hbar v_{\mathrm{b}}}
\end{aligned}
$$

The parameter $\zeta=\hbar v_{\mathrm{b}} \Delta /\left(2 \tilde{t}_{m_{1} m_{2}} \ell^{2}\right)$ measures the deviation from perfect energy and momentum conservation for tunneling pseudofermions. Requiring continuity of the wave function at $x= \pm L / 2$ yields a system of linear equations that we can use to find expressions for the transmission coefficients $t_{\uparrow}$ and $t_{\downarrow}$. Note that, due to chirality, continuity of the wave function is sufficient to ensure current conservation. As $t_{\uparrow}$ and $t_{\downarrow}$ turn out to be independent of energy $E$, the tunneling current is proportional to the density difference of the pseudospin components. Its explicit expression is

$$
I_{\mathrm{J}}^{(2)}=-\left|t_{\downarrow}\right|^{2} \sqrt{2} v_{\mathrm{b}} \bar{\varrho}_{\mathrm{b}} \operatorname{sgn}\left(\nu_{m_{1}}-\nu_{m_{2}}\right),
$$

with the transmission probability found from the above calculation as

$$
\left|t_{\downarrow}\right|^{2} \equiv T(L)=\frac{\sin ^{2}\left[\frac{\pi L}{L_{t}} \sqrt{1+\zeta^{2}}\right]}{1+\zeta^{2}} .
$$

Here $L_{t}=\pi \hbar v_{\mathrm{b}} / \tilde{t}_{m_{1} m_{2}}$ is a length scale set by the effective tunneling strength. From Eq. (36), we find the expression for the current through the line junction as a function of the externally applied voltage

$$
I_{\mathrm{J}}^{(2)}=\frac{\chi_{1}}{2 \pi \hbar} \frac{T(L)}{1+\frac{\nu_{m_{1}}+\nu_{m_{2}}}{2 \nu_{m_{1}} \nu_{m_{2}}} T(L)}\left(\mu_{1}-\mu_{3}\right) .
$$

With Eq. 41, we have the full solution of the transport problem for line junctions with effective filling factor $\tilde{\nu}=1 / 2$. A linear I-V characteristic is obtained, with a conductance oscillating as a function of junction length $L$ and magnetic field (through dependence on the parameter $\zeta$ ). Its maximum value, reached for $T(L) \rightarrow 1$ and given by

$$
G_{\mathrm{J}}^{\max }=\frac{e^{2}}{2 \pi \hbar} \min \left\{\nu_{m_{1}}, \nu_{m_{2}}\right\}
$$

is smaller than that of an adiabatic point contact between chiral fractional edge channels considered in Ref. [50], as should be expected.

\section{B. Case $\tilde{\nu}=1 / 4$ : Conductance oscillations as a function of transport voltage}

Refermionization yields an expression for the tunneling current in terms of the fictitious Dirac fermion $\psi_{\mathrm{b}}$,

$$
I_{\mathrm{J}}^{(4)}=\chi_{1} \frac{v_{\mathrm{t}}}{2} \int_{-\frac{L}{2}}^{\frac{L}{2}} d x\left\{\psi_{\mathrm{b}}^{\dagger} \partial_{x} \psi_{\mathrm{b}}^{\dagger}-\psi_{\mathrm{b}} \partial_{x} \psi_{\mathrm{b}}\right\}
$$

or the Majorana fermions that diagonalize the slow-mode Hamiltonian when $\Delta=0$ :

$$
I_{\mathrm{J}}^{(4)}=\frac{v_{\mathrm{t}}}{2} \int_{-\frac{L}{2}}^{\frac{L}{2}} d x\left\{\xi_{+}\left(-i \chi_{1} \partial_{x}\right) \xi_{-}+\xi_{-}\left(-i \chi_{1} \partial_{x}\right) \xi_{+}\right\}
$$

In close analogy with the chiral-tunneling problem encountered for $\tilde{\nu}=1 / 2$ and discussed in Sec. IVA above, we solve the transport problem through the line junction by considering the scattering of Dirac fermions $\psi_{\mathrm{b}}$ in terms of the Majorana normal modes. As in the Landauer-Büttiker formalism for transport, ${ }^{48.49}$ incoming scattering states are postulated for $\chi_{\mathrm{b}} x<-L / 2$, given in terms of a Majorana spinor notation as

$$
\left.\left(\begin{array}{c}
\xi_{+} \\
\xi_{-}
\end{array}\right)\right|_{\chi_{\mathrm{b}} x<-\frac{L}{2}}=\left(\begin{array}{c}
1 \\
i \chi_{\mathrm{b}}
\end{array}\right) e^{i x\left(\frac{\chi_{\mathrm{b}} E}{\hbar v_{\mathrm{b}}}-\frac{\Delta}{\ell^{2}}\right)}
$$

Outgoing scattering states exist for $\chi_{\mathrm{b}} x>L / 2$ and are superpositions of a Dirac-fermion state $\psi_{\mathrm{b}}$ and a Dirac 'hole' state $\psi_{\mathrm{b}}^{\dagger} .54$ 


$$
\left.\left(\begin{array}{c}
\xi_{+} \\
\xi_{-}
\end{array}\right)\right|_{\chi_{\mathrm{b}} x>\frac{L}{2}}=t_{-}\left(\begin{array}{c}
1 \\
i \chi_{\mathrm{b}}
\end{array}\right) e^{i x\left(\frac{\chi_{\mathrm{b}} E}{\hbar v_{\mathrm{b}}}-\frac{\Delta}{\ell^{2}}\right)}+t_{+}\left(\begin{array}{c}
1 \\
-i \chi_{\mathrm{b}}
\end{array}\right) e^{i x\left(\frac{\chi_{\mathrm{b}} E}{\hbar v_{\mathrm{b}}}+\frac{\Delta}{\ell^{2}}\right)} .
$$

In the line-junction region, a superposition of the two eigenstates with energy eigenvalue $E$ is realized:

$$
\left.\left(\begin{array}{c}
\xi_{+} \\
\xi_{-}
\end{array}\right)\right|_{|x|<\frac{L}{2}}=a\left(\begin{array}{c}
c_{k_{+}} \\
i \chi_{\mathrm{b}} s_{k_{+}}
\end{array}\right) e^{i k_{+} x}+b\left(\begin{array}{c}
i \chi_{\mathrm{b}} s_{k_{-}} \\
c_{k_{-}}
\end{array}\right) e^{i k_{-} x} .
$$

Their respective wave vectors are solutions of $E_{k_{ \pm}, \pm}=E$. Requiring continuity of the Majorana-spinor wave functions at the line-junction interfaces $x= \pm L / 2$, which simultaneously ensures current conservation for the chiral-fermion problem considered here, yields expressions for the transmission amplitudes $t_{ \pm}$. In contrast to the case $\tilde{\nu}=1 / 2$, the latter are energy dependent. The transport current through the line junction is given by

$$
I_{\mathrm{J}}^{(4)}=\frac{\chi_{1} \chi_{\mathrm{b}}}{2 \pi \hbar} \int_{0}^{2 \pi \hbar v_{\mathrm{b}} \bar{\varrho}_{\mathrm{b}}} d E\left|t_{+}(E)\right|^{2}
$$

hence we only need the general expression for $\left|t_{+}\right|^{2}$, which is found to be

$$
\left|t_{+}\right|^{2}=2 \frac{\left(\sqrt{1+r_{k_{+}}^{2}}-\left|r_{k_{+}}\right|\right)\left(\sqrt{1+r_{k_{-}}^{2}}+\left|r_{k_{-}}\right|\right)}{1+\left|r_{k_{+}}\right|\left|r_{k_{-}}\right|+\sqrt{1+r_{k_{+}}^{2}} \sqrt{1+r_{k_{-}}^{2}}} \sin ^{2}\left[\frac{1}{2}\left(k_{+}-k_{-}\right) L\right]
$$

Note that the energy dependence of $\left|t_{+}\right|^{2}$ is implicit through the dependence of $k_{ \pm}$on $E$.

Solving Eq. (48) in conjunction with Eq. (36) yields $I_{\mathrm{J}}^{(4)}$ as a function of the externally applied voltage $V=\left(\mu_{1}-\mu_{3}\right) / e$ and, hence, solves the transport problem through line junc-

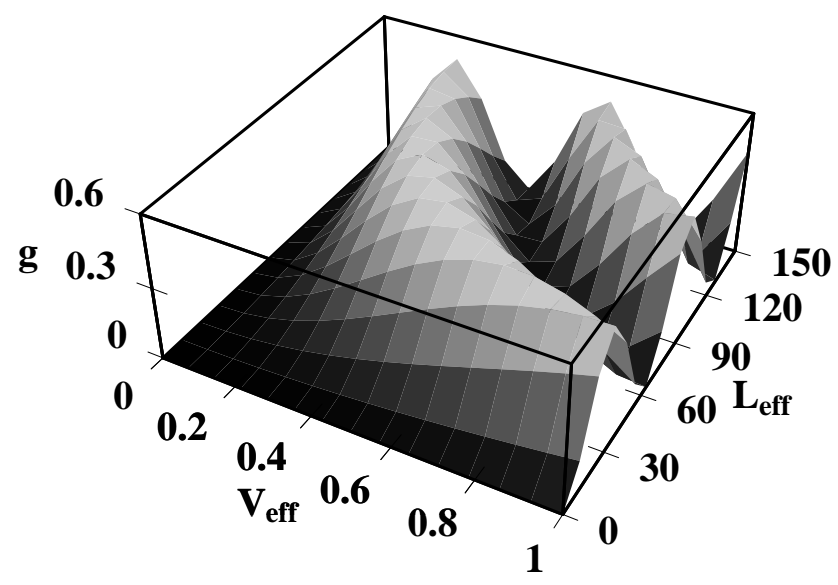

FIG. 2: Illustration of conductance oscillations. The dimensionless differential tunneling conductance $g=\frac{2 \pi \hbar}{e^{2}} \frac{d I}{d V}$ is plotted as a function of $V_{\text {eff }}=\frac{\epsilon \ell}{e} V$ and $L_{\text {eff }}=\frac{L}{\ell}$, according to Eq. 50. Our choice of units is motivated by the fact that magnetic length $\ell$ and Coulomb energy $e^{2} /(\epsilon \ell)$ are characteristic scales in the fractional-quantumHall regime. We assumed $e^{2} v_{\mathrm{t}} /\left(\epsilon \hbar v_{+} v_{-}\right)=0.1$ here but results for different values can be obtained by linear scaling of $V_{\text {eff }}$ or $L_{\text {eff. }}$. tions having effective filling factor $\tilde{\nu}=1 / 4$. While such a solution is accessible, even if only numerically, in the general case, it is instructive to look at the special situation where $\Delta=0$ and tunneling is weak. In this limit, we find for the differential tunneling conductance the expression

$$
e \frac{d I_{\mathrm{J}}^{(4)}}{d V}=\frac{e^{2}}{2 \pi \hbar} \frac{1}{4}\left\{1-\cos \left(\frac{v_{\mathrm{t}} L}{\hbar v_{+} v_{-}} e V\right)\right\}
$$

which is valid as long as the expression in curly brackets is smaller than unity. It exhibits an oscillatory dependence on both junction length and applied voltage, which we illustrate in Fig. 2 for a realistic set of parameters. The conductance oscillations as a function of $V$ are a direct consequence of the velocity splitting of the Majorana pseudofermions that are the quasiparticle excitations in the line junction. This signature behavior for $\tilde{\nu}=1 / 4$ will survive in the general case as long as the junction is narrow enough such that $\Delta<v_{\mathrm{t}} \ell^{2}|e V| /\left(\hbar v_{\mathrm{b}}^{2}\right)$. Its observation in real samples would provide strong evidence for the reality of the Majorana quasiparticles in QH line junctions, showing yet another example of how strongly-correlated condensed-matter systems exhibit features of relativistic quantum-field theories typically encountered in the realm of elementary-particle physics.

\section{SUMMARY AND CONCLUSIONS}

In this article, we provide a detailed theory of transport through line junctions formed between tunnel-coupled counter-propagating fractional-quantum-Hall edge channels having different filling factors of the Laughlin type. Strong 
correlations within the tunneling region result in a nontrivial spectrum of elementary excitations when the effective filling factor $\tilde{\nu}$ of the junction is equal to $1 / 2$ or $1 / 4$. An example for the former (latter) case is a line junction between edge channels having filling factor 1 and $1 / 3(1 / 5)$. Using bosonization and refermionization techniques, we map the original linejunction problem to a model of tunneling between noninteracting chiral spin-1/2 Dirac pseudofermions (for $\tilde{\nu}=1 / 2$ ) or a chiral spinless $\mathrm{p}$-wave superconductor with Majoranapseudofermion quasiparticles (for $\tilde{\nu}=1 / 4$ ). In both situations, we find the excitation spectrum and eigenstates of the line junction exactly. Together with an exact general relation, given by Eq. (36), between the externally applied chemicalpotential difference $\mu_{1}-\mu_{3}$ and intrinsic line-junction quantities, such as the total current $I_{\mathrm{J}}$, we can solve for the transport I-V characteristics. The full solution for the case $\tilde{\nu}=1 / 2$ is given by Eq. (41), together with Eq. 40b. It exhibits oscillations as a function of junction length that are similar to those found in tunnel-coupled quantum wires ${ }^{45,46,47}$ To calculate transport through junctions having $\tilde{\nu}=1 / 4$ in the most general case requires simultaneous numerical solution of Eqs. (48) and (36) for specific parameters realized in experiment. For the special situation of a narrow junction, analytical results are available. We give the differential tunneling con- ductance in Eq. [50, which shows oscillations as a function of transport voltage. This behavior is the signature of a nontrivial velocity splitting similar to spin-charge separation in interacting quasi-1D systems. Its observation would provide evidence of yet another mechanism for electron fractionalization in strongly correlated electron systems. Experiments in samples of 90 -degree bent ${ }^{21,24}$ or laterally separated ${ }^{22} 2 \mathrm{D}$ electron systems, suitably modified to reach the fractional quantum Hall regime, could be used to test our predictions.

\section{Acknowledgments}

Our work was supported in part by German Science Foundation (DFG) Grant No. ZU 116/1 and a German-Israeli Project Cooperation (DIP) of the German Ministry of Education and Research (BMBF). We enjoyed useful discussions with N. Andrei, C. de C. Chamon, P. Coleman, M. Grayson, V. Oganesyan, F. v. Oppen, Y. Oreg, S. L. Sondhi and A. Stern. E. S. wishes to thank the Department of Physics and Astronomy at Rutgers University for the hospitality during part of the time this work was carried out.
* Present and permanent address.

1 J. Voit, Rep. Prog. Phys. 57, 977 (1994).

2 S. Tomonaga, Prog. Theor. Phys. 5, 544 (1950).

3 J. M. Luttinger, J. Math. Phys. 4, 1154 (1963).

${ }^{4}$ D. C. Mattis and E. H. Lieb, J. Math. Phys. 6, 304 (1965).

5 K. D. Schotte and U. Schotte, Phys. Rev. 182, 479 (1969).

${ }^{6}$ A. Luther and I. Peschel, Phys. Rev. B 9, 2911 (1974).

7 P. Kopietz, Bosonization of Interacting Fermions in Arbitrary Dimensions (Springer, Berlin, 1997).

8 J. von Delft and H. Schoeller, Ann. Phys. (Leipzig) 7, 225 (1998).

${ }^{9}$ F. D. M. Haldane, J. Phys. C 14, 2585 (1981).

${ }^{10}$ S. Kagoshima, H. Nagasawa, and T. Sambongi, One-Dimensional Conductors (Springer, Berlin, 1988).

11 A. Yacoby, H. L. Störmer, N. S. Wingreen, L. N. Pfeiffer, K. W. Baldwin, and K. W. West, Phys. Rev. Lett. 77, 4612 (1996).

12 M. S. Dresselhaus, G. Dresselhaus, and P. C. Eklund, Science of Fullerenes and Carbon Nanotubes (Academic Press, San Diego, 1996).

13 A. H. MacDonald, in Mesoscopic Quantum Physics (Elsevier Science, Amsterdam, 1995), pp. 659-720, Proceedings of the 1994 Les Houches Summer School, Session LXI.

${ }^{14}$ R. E. Prange and S. M. Girvin, eds., The Quantum Hall Effect (Springer, New York, 1990), 2nd ed.

15 S. Das Sarma and A. Pinczuk, eds., Perspectives in the Quantum Hall Effects (Wiley, New York, 1997).

${ }^{16}$ B. I. Halperin, Phys. Rev. B 25, 2185 (1982).

17 X. G. Wen, Int. J. Mod. Phys. B 6, 1711 (1992).

18 A. M. Chang, L. N. Pfeiffer, and K. W. West, Phys. Rev. Lett. 77, 2538 (1996).

${ }^{19}$ M. Huber, M. Grayson, M. Rother, R. Deutschmann, W. Biberacher, W. Wegscheider, M. Bichler, and G. Abstreiter, Physica E 12, 125 (2001)

${ }^{20}$ M. Huber, M. Grayson, D. Schuh, M. Bichler, W. Biberacher, W.
Wegscheider, and G. Abstreiter, cond-mat/0309221

${ }^{21}$ M. Grayson, D. Schuh, M. Huber, M. Bichler and G. Abstreiter, cond-mat/0308576

22 W. Kang, H. L. Stormer, L. N. Pfeiffer, K. W. Baldwin, and K. W. West, Nature 403, 59 (1999).

23 A. Würtz, R. Wildfeuer, A. Lorke, E. V. Deviatov, and V. T. Dolgopolov, Phys. Rev. B 65, 075303 (2002).

${ }^{24}$ M. Grayson, D. Schuh, M. Bichler, G. Abstreiter, L. Hoeppel, J. Smet and K. von Klitzing, cond-mat/0308557

25 A. Mitra and S. M. Girvin, Phys. Rev. B 64, 041309 (2001).

${ }^{26}$ M. Kollar and S. Sachdev, Phys. Rev. B 65, 121304(R) (2002).

27 E.-A. Kim and E. Fradkin, Phys. Rev. B 67, 045317 (2003).

28 S. R. Renn and D. P. Arovas, Phys. Rev. B 51, 16832 (1995).

${ }^{29}$ C. L. Kane and M. P. A. Fisher, Phys. Rev. B 56, 15231 (1997).

30 J. D. Naud, L. P. Pryadko, and S. L. Sondhi, Nucl. Phys. B 565, 572 (2000).

31 J. D. Naud, L. P. Pryadko, and S. L. Sondhi, Phys. Rev. B 63, 115301 (2001).

${ }^{32}$ U. Zülicke and E. Shimshoni, Phys. Rev. Lett. 90, 026802 (2003).

${ }_{33}$ M. Stone, Bosonization (World Scientific, Singapore, 1994).

${ }^{34}$ U. Zülicke and A. H. MacDonald, Phys. Rev. B 54, 16813 (1996).

35 R. Shankar, Acta Phys. Pol. B 26, 1835 (1995).

36 A. O. Gogolin, A. A. Nersesyan, and A. M. Tsvelik, Bosonization (Cambridge University Press, Cambridge, UK, 1998).

37 U. Zülicke, Phys. Rev. Lett. 83, 5330 (1999).

${ }^{38}$ P. G. de Gennes, Superconductivity of Metals and Alloys (Addison-Wesley, Reading, MA, 1989).

39 N. Read and D. Green, Phys. Rev. B 61, 10267 (2000).

${ }^{40}$ M. Stone and R. Roy, cond-mat/0308034

41 A. M. Finkel'stein and A. I. Larkin, Phys. Rev. B 47, 10461 (1993).

42 M. Fabrizio and A. Parola, Phys. Rev. Lett. 70, 226 (1993).

${ }^{43}$ U. Zülicke and M. Governale, Phys. Rev. B 65, 205304 (2002). 
44 I. Safi, Eur. Phys. J. B 12, 451 (1999).

45 J. A. del Alamo and C. C. Eugster, Appl. Phys. Lett. 56, 78 (1990).

46 O. E. Raichev and P. Vasilopoulos, J. Phys.: Condens. Matter 12, 6859 (2000).

${ }^{47}$ D. Boese, M. Governale, A. Rosch, and U. Zülicke, Phys. Rev. B 64, 085315 (2001).

48 R. Landauer, IBM J. Res. Dev. 1, 223 (1957).

49 M. Büttiker, IBM J. Res. Dev. 32, 317 (1988).

${ }^{50}$ D. B. Chklovskii and B. I. Halperin, Phys. Rev. B 57, 3781 (1998).

${ }^{51}$ A description in terms of nonchiral phase fields would only be possible at the special (but unphysical) point in parameter space where $U=2 \pi \hbar\left(\nu_{m_{2}}-\nu_{m_{1}}\right)\left(v_{\mathrm{F} 1}-v_{\mathrm{F} 2}\right)$.

52 As it would be unphysical to neglect the velocity $v_{\mathrm{b}}$ of the slow normal mode, all terms to first order in the small quantities have to be kept for consistency. We thank C. Chamon for focusing our attention on this point. Note that, for this reason, the normal modes of $H_{\mathrm{LL}}$ are only approximately, and not exactly, equal to the familiar charged and neutral modes defined, e.g., in Ref. [32].

53 This is due to the power-law system-size dependence of the normalization factors $z_{m_{j}}$. See, e.g., J. M. Kinaret, Y. Meir, N. S. Wingreen, P. A. Lee, and X. G. Wen, Phys. Rev. B 46, 4681 (1992).

54 This is not surprising, as the refermionized slow-mode Hamiltonian $H_{\mathrm{b}}^{(4)}$ in the representation of Dirac fermions is analogous to the Bogoliubov-de Gennes equation for a p-wave superconductor. See Eq. 16 and remarks below. 